\title{
Estado nutricional en una población pediátrica de una zona rural de Cusco, Perú
}

\author{
Nutritional status in a pediatric population in a rural area from Cusco, Peru
}

\section{Sr. Editor:}

La desnutrición infantil es un problema de salud pública en el mundo, especialmente en países en vías de desarrollo (1). En el Perú la desnutrición crónica afecta a un porcentaje significativo de la población especialmente niños en zonas rurales, en la sierra y a los que se encuentran en el quintil inferior de extrema pobreza (2). Con el objetivo de determinar el estado nutricional en una población pediátrica de una comunidad rural de Cusco (Perú), se realizó un estudio transversal, descriptivo en la comunidad de Marcaconga ubicada a 3763 msnm, distrito Sangarará, provincia de Acomayo, departamento Cusco, calificada en el quintil I de pobreza (3). Se realizó la revisión de las historias clínicas de la totalidad de preescolares y escolares; pertenecientes a la posta de salud de dicha comunidad. Se incluyó en el estudio a niños y niñas con edades comprendidas entre 3 y 11 años de edad que residían como mínimo un año en la comunidad y que contaban con registros actualizados por lo menos de un mes de antigüedad de las variables a estudiar. Se excluyeron del estudio a los niños y niñas con antecedentes patológicos según su historia clínica.

Se consideraron las variables sexo, edad, talla, índice de masa corporal (IMC). Se dividió en dos grupos etarios: de 36 meses y menores de 5 años calificados "preescolares" y de 5 a 11 años calificados "escolares". Para la determinación del estado nutricional en los preescolares se utilizó peso, talla, sexo y edad para ubicarlos en la curva de peso para la talla, talla para la edad y peso para la edad, según la puntuación "Z" de la OMS (0-5 años) con la cual se estableció la presencia de desnutrición aguda, desnutrición crónica o desnutrición global. En el caso de los escolares se utilizó el peso, talla, edad, IMC y el sexo para ubicarlos en las curvas de IMC para la edad y talla para la edad según la puntuación "Z" de la OMS (5 a 19 años) (4). Con ello se estableció si el escolar presentaba delgadez severa, delgadez normal, sobrepeso y obesidad. Se realizó estadística descriptiva, en las variables categóricas se determinó frecuencias absoluta y relativa; y en las variables numéricas, mediana y rango intercuartílico. La ejecución del estudio fue aprobada por el Instituto de Investigación Médica de la Universidad Nacional San Antonio Abad del Cusco, Perú y la posta de salud de la mencionada comunidad.

Se incluyó en el estudio los datos de 378 niños. El $51,1 \%$ fue del sexo masculino y el $71,2 \%$ escolares. La mediana de edad fue 7 años (rango intercuartílico 5 a 8 años). En cuanto al estado nutricional, $62(56,9 \%)$ preescolares presentaron desnutrición crónica y $10,1 \%$ desnutrición aguda. El 94,8\% de los escolares tuvo estado nutricional normal (Tabla 1).

Tabla 1. Estado de nutrición en preescolares y escolares de la Comunidad de Marcaconga, Cusco, Perú (2014).

\begin{tabular}{lcr}
\hline Estado de Nutrición & n & $\mathbf{( \% )}$ \\
\hline Preescolares (36 meses - < 5 años) & & \\
Desnutrición aguda & 11 & 10,1 \\
Desnutrición crónica & 62 & 56,9 \\
Desnutrición global & 3 & 2,7 \\
Normal & 33 & 30,3 \\
Escolares (5 años - 11 años) & & \\
Delgadez & 1 & 0,4 \\
Normal & 255 & 94,8 \\
Sobrepeso & 9 & 3,3 \\
Obesidad & 4 & 1,5 \\
\hline
\end{tabular}


En el Perú, la desnutrición constituye un problema de salud pública que aún sigue siendo motivo de estudio por el impacto económico que genera (5), por el riesgo de predisposición a infecciones, por el impacto en el desarrollo cognitivo de los niños a largo plazo, pudiendo establecerse diferencias sociales por la distribución heterogénea de la población peruana (6).

Los niños preescolares presentaron desnutrición crónica en más de la mitad de los casos, hallazgo similar al de un estudio realizado en Apurímac en una población similar a la nuestra (7). Así mismo, se evidenció adecuado estado nutricional en la mayoría de niños escolares. Esto podría deberse a la implementación de los programas de alimentación complementaria establecidos por el gobierno peruano, los cuales tendrían un impacto positivo con la población beneficiaria asociándose con una disminución de la desnutrición en la población escolar (8); pero no en la población preescolar siendo este el grupo aún vulnerable a presentar desnutrición. Sin embargo, podría cambiar en los próximos años por los cambios alimenticios a la cual está siendo susceptible la población en general y de alguna forma reflejaría los mínimos casos de sobrepeso y obesidad encontrados en dicha población (9).

Recomendamos realizar estudios de seguimientos en poblaciones rurales en pobreza y extrema pobreza para ver la efectividad de los programas sociales; así como, para evidenciar la transición del estado nutricional en poblaciones rurales, de esta forma reorientar las políticas de salud para lograr adecuadas estrategias en la detección oportuna y tratamiento adecuado de esta enfermedad.

\section{Cristhiam Monterroso-Cusihuallpa ${ }^{1, a}$, Evelina Andrea Rondón-Abuhadba ${ }^{1, b}$, Noé Atamari- Anahuii ${ }^{1, a}$, Jhonatan Julio Llallicuna-Quiñones ${ }^{1, \mathrm{c}}$, María Estéfany Tupayachi-Atapaucar ${ }^{1, c}$}

\section{Correspondencia:}

Noé Atamari-Anahui

Dirección: Calle Los Geranios B-2 San Sebastián, Cusco, Perú

Teléfono: (51) 984140761

Correo electrónico: noe.atamari@gmail.com

\section{REFERENCIAS BIBLIOGRÁFICAS}

1. Black RE, Victora CG, Walker SP, et al. Maternal and child undernutrition and overweight in lowincome and middle-income countries. Lancet. 2013; 382(9890):427-51.

2. Sobrino M, Gutiérrez C, Cunha AJ, Dávila M, Alarcón J. Child malnutrition in children under 5 years of age in Peru: trends and determinants. Rev Panam Salud Pública. 2014; 35(2):104-12.

3. Dirección Regional de Salud Cusco. Análisis de la Situación de Salud: Provincia de Acomayo. Cusco: Red de Servicios de Salud Cusco Sur; 2015. Citado 9 de enero de 2018) Disponible en: http://www. diresacusco.gob.pe/ASISprov/acomayo.pdf

4. Onis M, Onyango AW, Borghi E, Siyam A, Nishida C, Siekmann J. Development of a WHO growth reference for school-aged children and adolescents. Bull World Health Organ. 2007; 85(9):660-7.

5. Alcázar L, Ocampo D, Huamán-Espino L, Aparco P. Impacto económico de la desnutrición crónica, aguda y global en el Perú. Rev Peru Med Exp Salud Publica. 2013; 30(4):569-74.

6. Martínez R, Fernández A. El costo del hambre: impacto social y económico de la desnutrición infantil en el Estado Plurinacional de Bolivia, el Ecuador, Paraguay y el Perú. Santiago de Chile: CEPAL; 2009.

7. Andrissi L, Mottini G, Sebastiani V, Boldrini L, Giuliani A. Dietary habits and growth: an urban/rural comparison in the Andean region of Apurimac, Peru. Ann Dell Istituto Super Sanità. 2013; 49(4):340-6.

8. Pérez-Lu JE, Cárcamo C, Nandi A, Kaufman JS. Health effects of «Juntos», a conditional cash transfer programme in Peru. Matern Child Nutr. 2017;13(3):00. doi: $10.1111 / \mathrm{mcn} .12348$

9. Torres-Roman JS, Azañedo D, Ruiz EF, Avilez JL, Málaga G. La doble carga de la desnutrición: una amenaza para la niñez peruana. Gac Sanit. 2017; 31(4):359-60. 